JOURNAL OF FOOT

AND ANKLE RESEARCH

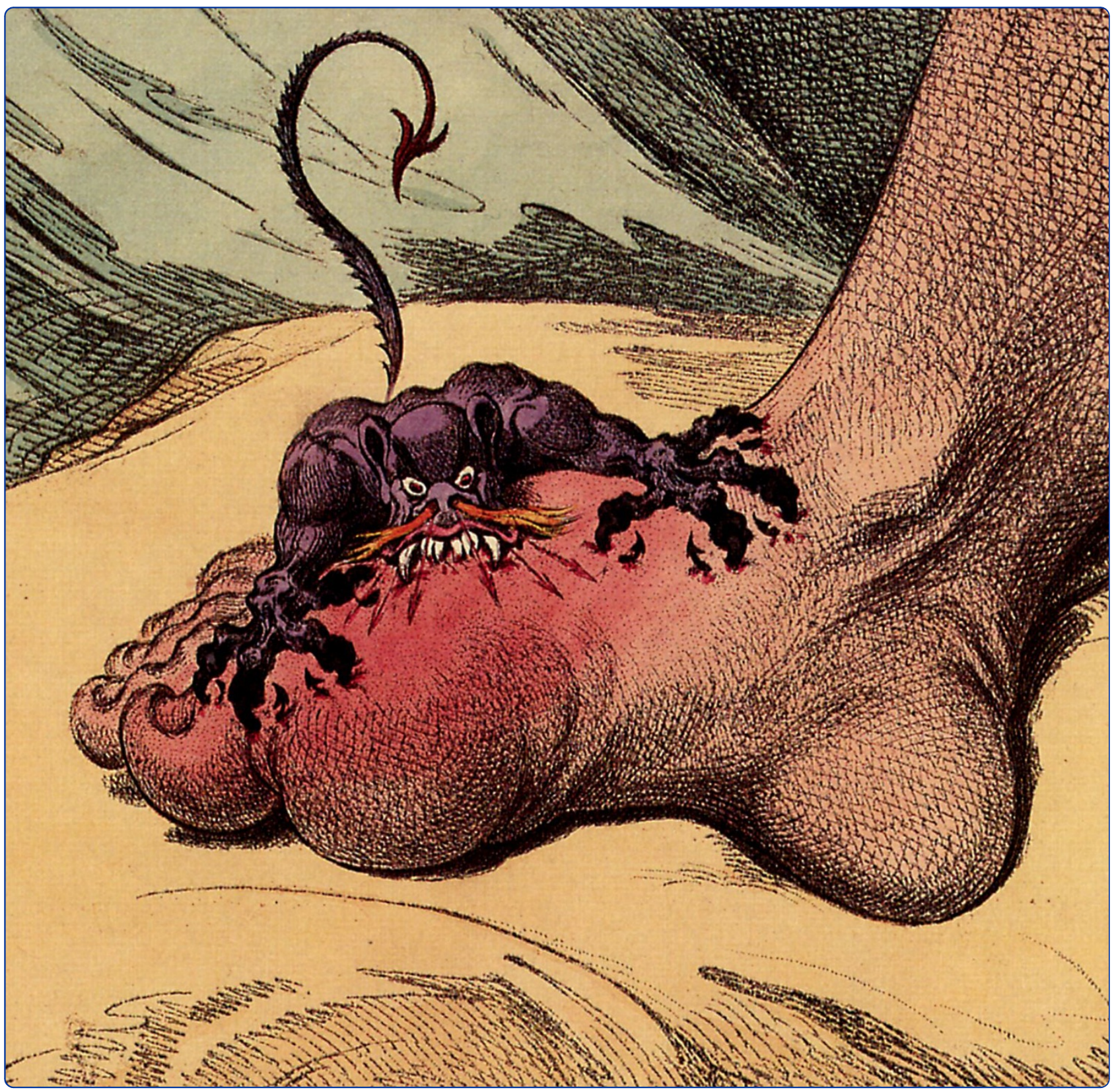

\title{
Revisiting the pathogenesis of podagra: why does gout target the foot?
}

Roddy 


\title{
Revisiting the pathogenesis of podagra: why does gout target the foot?
}

\author{
Edward Roddy
}

\begin{abstract}
This invited paper provides a summary of a keynote lecture delivered at the 2011 Australasian Podiatry Conference. Gout is the most prevalent inflammatory arthropathy. It displays a striking predilection to affect the first metatarsophalangeal joint as well as joints within the mid-foot and ankle. A number of factors are known to reduce urate solubility and enhance nucleation of monosodium urate crystals including decreased temperature, lower $\mathrm{pH}$ and physical shock, all of which may be particularly relevant to crystal deposition in the foot. An association has also been proposed between monosodium urate crystal deposition and osteoarthritis, which also targets the first metatarsophalangeal joint. Cadaveric, clinical and radiographic studies indicate that monosodium urate crystals more readily deposit in osteoarthritic cartilage. Transient intra-articular hyperuricaemia and precipitation of monosodium urate crystals is thought to follow overnight resolution of synovial effusion within the osteoarthritic first metatarsophalangeal joint. The proclivity of gout for the first metatarsophalangeal joint is likely to be multi-factorial in origin, arising from the unique combination of the susceptibility of the joint to osteoarthritis and other determinants of urate solubility and crystal nucleation such as temperature and minor physical trauma which are particularly relevant to the foot.
\end{abstract}

\section{Background}

Gout is a true crystal deposition disease in which all clinical manifestations are considered to be directly attributable to the presence of monosodium urate (MSU) crystals. It is one of the most prevalent inflammatory arthropathies with a prevalence of approximately $1.4 \%$, and is the most common inflammatory arthropathy in men [1]. Both the prevalence and incidence of gout appear to be rising [2]. The primary risk factor for the development of gout is elevation of serum uric acid (urate) levels, or hyperuricaemia. As uric acid levels rise and exceed the physiological saturation threshold of uric acid in body tissues, formation and deposition of MSU crystals occurs in and around joints.

The propensity of gout for the foot was recognised by the ancient Greeks who referred to it as podagra, literally "foot-grabber" [3]. The name "gout" derives from humoral theory and the Latin word gutta or "drop", podagra being thought to arise as a result of the bodily humours falling to the affected body part. Although our current understanding of the pathogenesis of gout is

Correspondence: e.roddy@cphc.keele.ac.uk

Arthritis Research UK Primary Care Centre, Primary Care Sciences, Keele University, Keele, UK dramatically distant from humoral theory, these observations concerning the intimate relationship between gout and the foot have been reinforced over the centuries and continue today. This review will consider the ways in which gout affects the foot and discuss potential mechanisms underlying this relationship.

\section{Clinical presentation of gout and involvement of the foot}

After an often prolonged period of asymptomatic hyperuricaemia, the initial manifestation of gout is usually an acute attack of synovitis affecting a single peripheral joint, most commonly the first metatarsophalangeal joint (MTPJ). Other commonly affected joints include the mid-tarsal joints, ankles, knees, fingers, wrists and elbows (Figure 1). Such attacks are characterised by sudden onset of excruciating joint pain, typically taking less than 24 hours from symptom onset to reach peak intensity, with associated joint swelling, overlying erythema and exquisite tenderness to touch. Although acute gout should be treated rapidly with a non-steroidal antiinflammatory drug (NSAID) or colchicine, it usually resolves completely over a period of two to three weeks even without treatment. A variable period of time then 


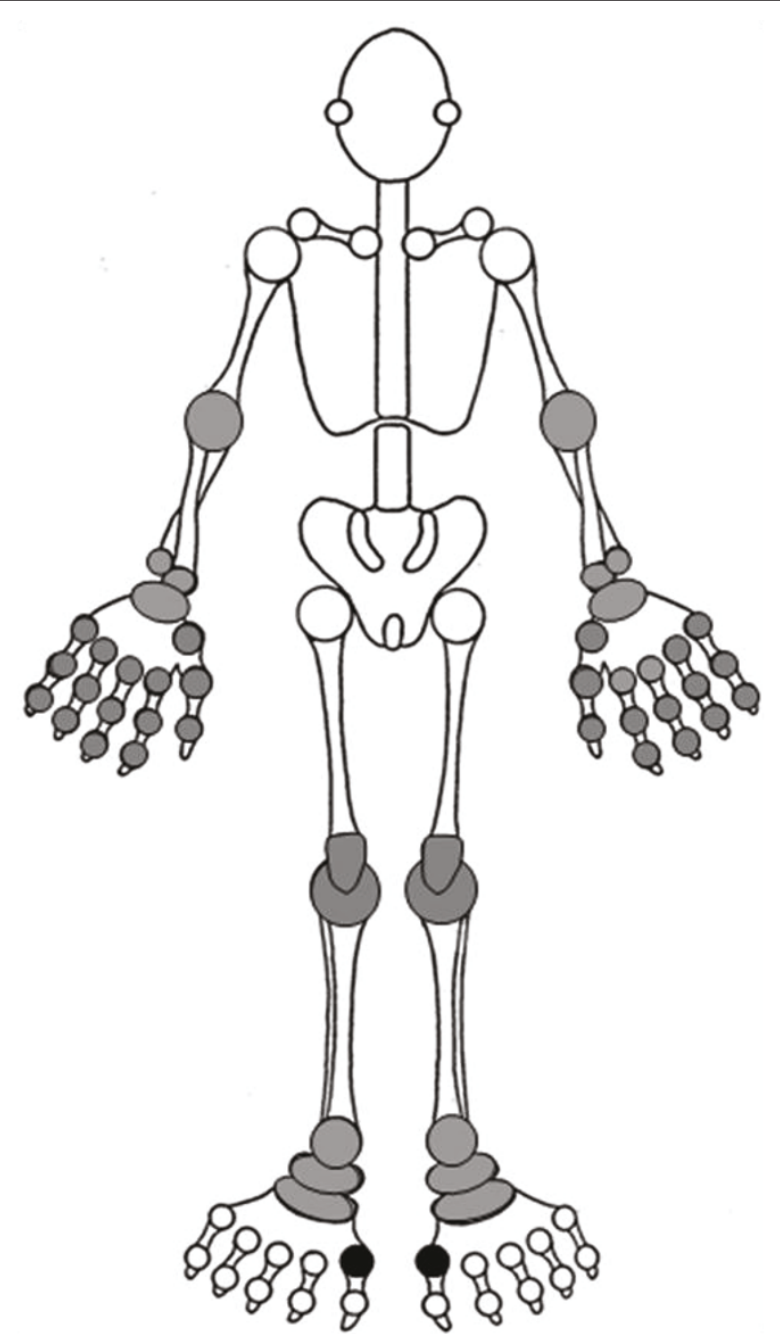

Figure 1 Distribution of joints typically affected by gout (reproduced with the permission of the author and the Royal College of General Practitioners: Roddy E, Doherty M. Gout. In: RCGP Guide to MSK Disorders in Primary Care. Ed: Warburton L (in press)).

elapses until the patient experiences a further attack (the "intercritical period"). With time, attacks may increase in severity and frequency, involve different joint sites, and may become oligo- or polyarticular. Eventually, without treatment, the patient may develop chronic tophaceous gout, characterised by chonic pain and stiffness, joint damage and erosive arthropathy, and clinically evident subcutaneous nodular deposits of MSU crystals (tophi) which can occur at the toes, Achilles' tendons, pre-patellar tendons, fingers, olecranon processes, and less commonly, the ears (Figure 2).

Gout displays a striking tendency to affect the foot, in particular the first MTPJ. The initial attack of gout affects the first MTPJ in 56-78\% of patients [4-7] and the joint is involved at some point in the course of disease in 59-89\% [4,6,8-10]. Fewer studies report the frequency of involvement of other joints. However, mid-foot and ankle involvement occurs in $25-50 \%$ and $18-60 \%$ of patients respectively $[5,8,9]$. In contrast, the upper limb is involved in $13-46 \%[4,6,8,10]$ and the finger interphalangeal joints in only 6-25\% [5,8,9].

Sub-clinical involvement in the foot also appears to be common-place. MSU crystal deposits have been observed in synovial fluid aspirated from first MTPJs that have never been affected by an acute attack of gout $[11,12]$. Furthermore, a study which examined the first MTPJs of 39 males with gout using high resolution ultrasonography found erosions to be present in $45 \%$ of 22 first MTPJs that had never been affected by acute gout [13].

Gout has a number of chronic manifestations which are easily recognisable as such including tophaceous deposits and a characteristic erosive arthropathy. However, it is also associated with a number of other less specific foot problems. Perhaps not surprisingly given the frequency of first MTPJ involvement, hallux valgus is a common finding. In a community-based casecontrol study, hallux valgus was found in $41 \%$ of gout suffers compared to $25 \%$ of age- and gender-matched control subjects (odds ratio (OR) 2.10, 95\% confidence interval (CI) 1.39 to 3.18 , adjusted for body mass index (BMI) and use of diuretics) [14]. Big toe pain occurring on most days for at least a month within the last year was reported by $16 \%$ of those with gout compared to $6 \%$ of controls (adjusted OR 2.94, 95\% CI 1.62 to 5.34). Given the striking predilection of gout for the foot, there has been surprisingly little work examining the influence of gout on foot function, gait and plantar pressure distributions. A recent study compared functional and biomechanical foot characteristics between 25 patients with chronic gout and 25 age- and gender-matched control subjects with no history of gout [15]. Patients with chronic gout were found to have slower walking velocity, reduced step and stride length, reduced peak plantar pressure under the hallux, and higher mid-foot pressure-time integrals compared to controls. The authors postulate that gait pattern is altered in chronic gout in an attempt to offload the first MTPJ thereby reducing pain. Further studies are necessary to explore these observations in more detail and examine the contribution of chronic pain in the great toe, hallux valgus, obesity and osteoarthritis (OA) to gait patterns in patients with gout.

\section{Factors influencing crystal deposition}

Gout is one of the best understood inflammatory arthropathies. Clinical features can be easily understood and interpreted in the context of a clearly elucidated pathogenetic process. Specific risk factors such as genetics, dietary factors, co-morbidity and its treatment lead to 

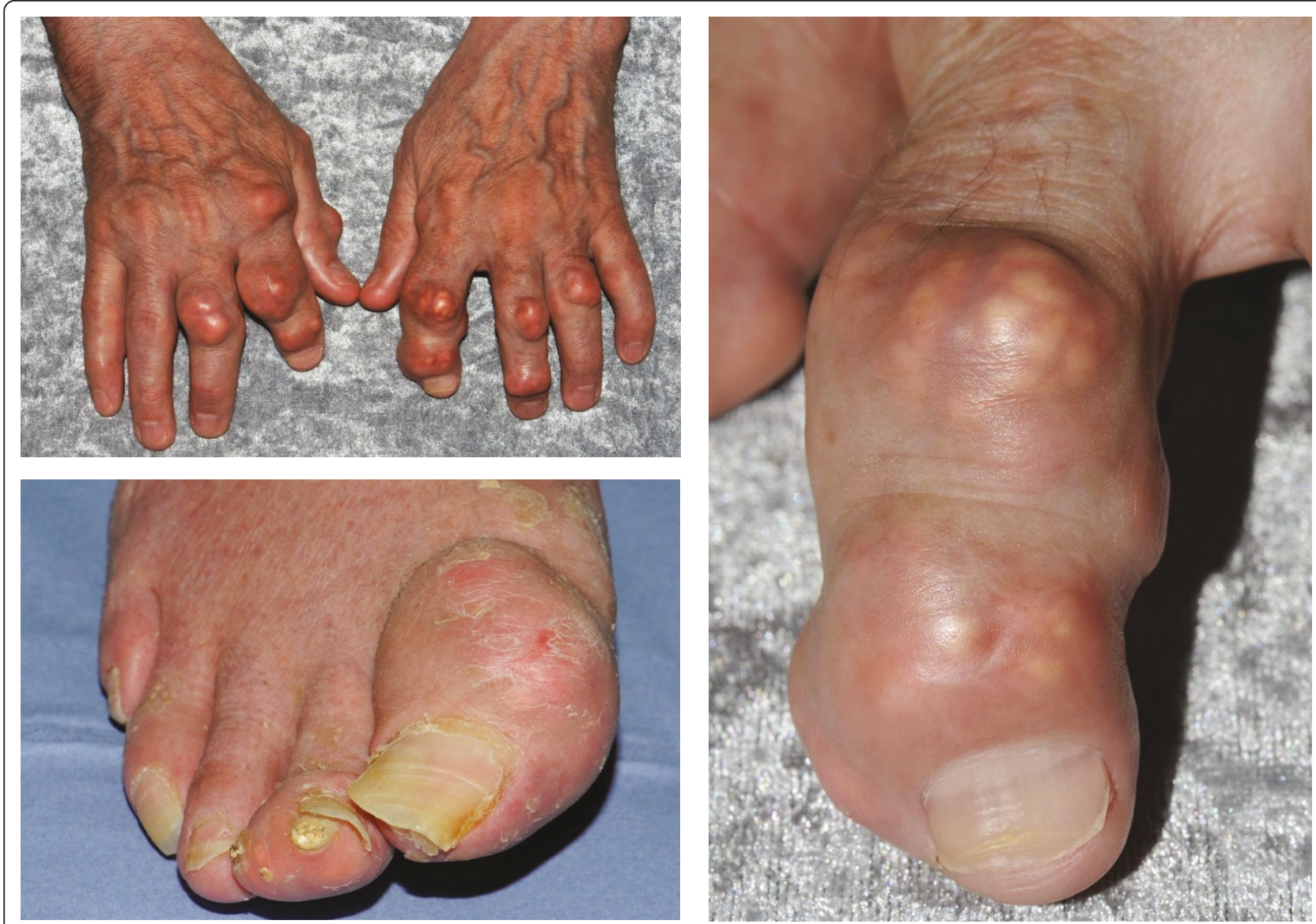

Figure 2 Tophaceous gout affecting the right great toe and finger interphalangeal joints. Note the asymmetrical swelling and yellowwhite discolouration.

hyperuricaemia and subsequently MSU crystal formation occurs $[16,17]$. Crystals are then shed into the joint and activate the inflammatory cascade via the NALP3 inflammasome $[18,19]$. Hence, any explanation of why gout targets the foot must link these pathological processes to the specific anatomical, functional, and disease characteristics of the foot (Figure 3).

\section{Temperature}

As described above, gout tends to affect distal peripheral joints, not only in the foot but also in the upper limb, with central axial joints such as the shoulders, hips and spine only rarely affected. The solubility of urate decreases with reducing temperature $[20,21]$ enhancing nucleation of MSU crystals, that is, the "birth" of new crystals. Reduced solubility of urate at lower temperatures has therefore been suggested to account for the occurrence of gout at cooler distal joints such as the foot-ankle complex. However, this theory does not account for the preference of gout for the first MTPJ ahead of the great toe interphalangeal (IP) joint or the lesser MTPJs.

\section{Trauma and $\mathrm{pH}$}

A further well-recognised clinical feature of gout is the tendency of an acute attack to be precipitated by physical trauma such as stubbing the toe or following physical activity. Enhanced MSU crystal nucleation has been reported in vitro following mechanical agitation of solutions supersaturated with sodium urate [22]. The same authors demonstrated that nucleation is also potentiated by both acidification and addition of calcium ions. Lowering of $\mathrm{pH}$ has a direct action on MSU crystal nucleation but also enhances nucleation by increasing calcium ion activity. Whilst their observations concerning mechanical agitation provide evidence that a physical shock can directly lead to MSU crystal nucleation, the authors hypothesised that local trauma indirectly enhances crystal nucleation by lowering synovial pH [22]. Hence, the susceptibility of the foot to physical trauma might also help to explain the predilection of gout for the foot.

\section{Cartilage damage and osteoarthritis}

More recently, the deposition of MSU and calcium pyrophosphate dihydrate (CPPD) crystals in areas of 


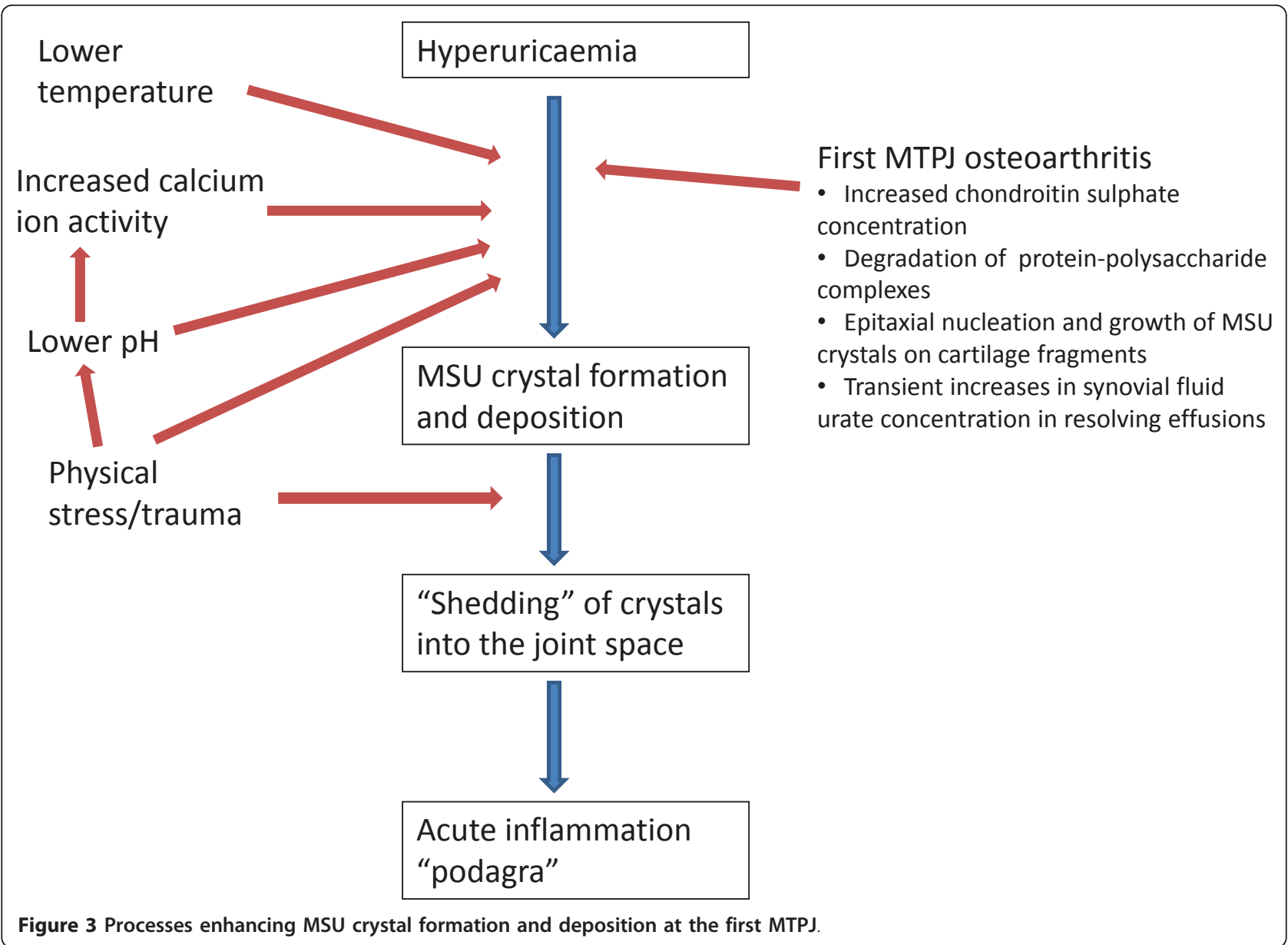

cartilage damage has been described in a cadaveric study which examined 7855 adult human tali from 4007 donors [23]. Crystal deposits, both MSU and CPPD, were an uncommon finding, being present in specimens from only $5 \%$ of donors. However, where seen, crystal deposits were usually found within or adjacent to a cartilage lesion. Only $8 \%$ of tali with crystal deposits had no gross evidence of cartilage degeneration. Cartilage lesions tended to be located at sites of biomechanical stress such as the articulation of the margin of the trochlea with the tibia or fibula or where apposition with anterior tibial osteophytes was thought to have occurred. In a separate study, the epitaxial nucleation and growth of MSU crystals was observed to occur on fragments of articular cartilage [24]. Thus there appears to be a relationship between cartilage lesions and the anatomical location of MSU crystal deposition.

In support of these observations, clinical and radiographic evidence exists of an association between gout and OA. Several case reports and small case series describe the occurrence of acute attacks of gout and/or tophi at first MTPJs and finger distal interphalangeal
(DIP) joints also affected by OA [25-30]. A Polish hospital-based study of 262 patients with gout found an association of gout and radiographic OA at the first MTPJs, tarsal joints and knees [31]. A more recent study of 164 patients with gout recruited from primary care found a very strong association between joints that had previously been the site of an acute attack of gout and evidence of OA on clinical examination (OR 7.94, $95 \%$ CI 6.27 to 10.05 , adjusted for age, gender, BMI and diuretic use) [8]. Significant associations were seen between acute attacks of gout and the presence of clinical OA at the first MTPJs, mid-foot, knee and finger DIP joints.

\section{Why are gout and osteoarthritis associated?}

The observations outlined above that MSU crystals tend to deposit at sites of cartilage damage and that clinical and radiographic evidence exists of an association between gout and OA lead to the important question of the mechanism by which gout and OA might be associated. There are three possible explanations for this association. 
Firstly, does an association exist between the disease states of gout and nodal generalised OA? These two conditions share the common risk factor of obesity $[32,33]$. In a related study to the primary care study described above [8], generalized nodal OA, defined as the presence of Heberden's or Bouchard's nodes on at least two digits in each hand [34], was no more commonplace in subjects with gout than age-and gendermatched community controls but, as discussed above, hallux valgus and self-reported knee and big toe pain were more frequent in those with gout [14]. Although this case-control study was underpowered, these findings do not suggest that an association exists between the disease states of gout and generalised OA.

The second and third explanations are related and concern the hypothesis that the association of gout and OA occurs at local joint sites and relates to the co-location of MSU crystal deposits and cartilage lesions. Specifically, they question the direction of this association, namely, does the presence of osteoarthritic cartilage predispose to the local formation and deposition of MSU crystals or do MSU crystals themselves initiate and progress cartilage damage? Evidence to support the deposition of MSU crystals in osteoarthritic cartilage rather than MSU crystals leading to cartilage damage arises from two sources. Although the primary care study described above was cross-sectional, making it difficult to infer causality, the strength of the association between involvement of gout and OA at individual joint sites did not increase with longer duration of gout [8]. A further insight into the direction of association between MSU crystal deposition and OA is provided by a recent study which examined the relationship between synovial fluid uric acid levels and the radiographic severity of knee OA [35]. Although synovial fluid uric acid was found to correlate with baseline knee OA severity, it was not associated with change in OA severity over 3 years. These two observations do not suggest that the association between the occurrence of gout and OA at individual joint sites is due to MSU crystal-initiated joint damage. Furthermore, certain properties of the osteoarthritic joint are thought to influence urate solubility and predispose to local MSU crystal disposition [36]. Increased concentrations of chondroitin sulphate and degradation of protein-polysaccharide complexes found within articular cartilage have been shown to reduce urate solubility and lead to the precipitation and growth of MSU crystals [37-39]. However, it is also possible that the association between MSU crystal deposition and $\mathrm{OA}$ is bi-directional whereby existing osteoarthritic change predisposes to local formation and deposition of MSU crystals which then initiate further cartilage damage.

\section{Why does gout target the first metatarsophalangeal joint?}

The studies discussed above provide clear evidence of an association between MSU crystal deposition and OA. Whilst further studies are required to definitively answer the questions of direction of association and causality, it appears that MSU crystals more readily deposit in osteoarthritic cartilage and that the presence of OA influences the distribution of joints affected by gout. However, OA cannot solely explain the typical distribution of joints affected by gout, as many joints commonly affected by OA such as the knees, finger IP joints, and hips are less frequently affected by gout than the first MTPJ, and other target joints for gout such as the ankle, wrist and elbow are infrequent sites for primary OA. Is it plausible therefore that the relationship between MSU crystal deposition and OA is of more relevance for the first MTPJ than other joint sites?

The first MTPJ is certainly targeted by OA although foot $\mathrm{OA}$ is under-studied in comparison to other commonly affected sites such as the hand and knee. A recent systematic review of population-based epidemiological studies found that the estimated prevalence of radiographic OA at the first MTPJ may be as high as $39 \%$ in middle-aged to older adults [40]. Simkin proposed a model to explain the clinical observations that acute attacks of gout are commonly precipitated by physical stress and occur overnight, based upon the cooccurrence of gout and OA at the first MTPJ [41]. In this model, a synovial effusion develops in an osteoarthritic first MTPJ during the day and subsequently resolves when the joint is rested overnight. Synovium is more permeable to water than urate and hence, as the effusion resolves, water leaves the joint more rapidly than urate. This results in a transient increase in the synovial fluid urate concentration which leads to precipitation of MSU crystals if the saturation threshold of urate is exceeded. As discussed above, MSU crystal formation and deposition will be further potentiated in the osteoarthritic first MTPJ by impaired urate solubility and enhanced crystal nucleation arising from factors relating to the anatomical location of the first MTPJ namely lower distal temperature and physical stress [20-22], and those relating to OA namely increased concentrations of chondroitin sulphate, degradation of protein-polysaccharide complexes, and epitaxial MSU crystal nucleation and growth on cartilage fragments [24,37-39] (Figure 3).

\section{Conclusion}

The striking predilection of gout for the first MTPJ appears to be multi-factorial in origin and arises from the unique combination of the susceptibility of the joint 
to $\mathrm{OA}$ and local anatomical considerations of temperature, minor physical trauma and biomechanical stress, leading to ideal conditions for MSU crystal formation and deposition in predisposed hyperuricaemic individuals, manifesting as clinical gout.

\section{Acknowledgements}

The author would like to thank Dr George Peat for helpful comments on the manuscript. The author is supported by an Arthritis Research UK Primary Care Centre Grant (18139).

\section{Competing interests}

The author declares that they have no competing interests.

Received: 21 April 2011 Accepted: 13 May 2011 Published: 13 May 2011

\section{References}

1. Annemans L, Spaepen E, Gaskin M, Bonnemaire M, Malier V, Gilbert T, Nuki G: Gout in the UK and Germany: prevalence, comorbidities and management in general practice 2000-2005. Ann Rheum Dis 2008, 67:960-966.

2. Roddy E, Zhang W, Doherty M: The changing epidemiology of gout. Nat Clin Pract Rheumatol 2007, 3:443-449.

3. Porter R, Rousseau GS: Gout The Patrician Malady New Haven and London: Yale University Press; 1998.

4. Puig JG, Michan AD, Jimenez ML, Perez de Ayala C, Mateos FA, Capitan CF, de Miguel E, Gijon JB: Female gout. Clinical spectrum and uric acid metabolism. Arch Intern Med 1991, 151:726-732.

5. Mijiyawa M: Gout in patients attending the rheumatology unit of Lome Hospital. Br J Rheumatol 1995, 34:843-846.

6. Lally EV, Ho G Jr, Kaplan SR: The clinical spectrum of gouty arthritis in women. Arch Intern Med 1986, 146:2221-2225.

7. Klemp P, Stansfield SA, Castle B, Robertson MC: Gout is on the increase in New Zealand. Ann Rheum Dis 1997, 56:22-26.

8. Roddy E, Zhang W, Doherty M: Are joints affected by gout also affected by osteoarthritis? Ann Rheum Dis 2007, 66:1374-1377.

9. Grahame R, Scott JT: Clinical survey of 354 patients with gout. Ann Rheum Dis 1970, 29:461-468.

10. Hall AP, Barry PE, Dawber TR, McNamara PM: Epidemiology of gout and hyperuricemia. A long-term population study. Am J Med 1967, 42:27-37.

11. Weinberger A, Schumacher HR, Agudelo CA: Urate crystals in asymptomatic metatarsophalangeal joints. Ann Intern Med 1979, 91:56-57.

12. Rouault T, Caldwell DS, Holmes EW: Aspiration of the asymptomatic metatarsophalangeal joint in gout patients and hyperuricemic controls. Arthritis Rheum 1982, 25:209-212.

13. Wright SA, Filippucci E, McVeigh C, Grey A, McCarron M, Grassi W, Wright GD, Taggart AJ: High-resolution ultrasonography of the first metatarsal phalangeal joint in gout: a controlled study. Ann Rheum Dis 2007, 66:859-864.

14. Roddy E, Zhang W, Doherty M: Gout and nodal osteoarthritis: a casecontrol study. Rheumatology (Oxford) 2008, 47:732-733.

15. Rome K, Survepalli D, Sanders A, Lobo M, McQueen FM, McNair P, Dalbeth N: Functional and biomechanical characteristics of foot disease in chronic gout: A case-control study. Clin Biomech (Bristol, Avon) 2011, 26:90-94.

16. Merriman TR, Dalbeth N: The genetic basis of hyperuricaemia and gout. Joint Bone Spine 2011, 78:35-40

17. Roddy E, Doherty M: Gout. Epidemiology of gout. Arthritis Res Ther 2010, $12: 223$.

18. Martinon F, Petrilli V, Mayor A, Tardivel A, Tschopp J: Gout-associated uric acid crystals activate the NALP3 inflammasome. Nature 2006, 440:237-241.

19. Petrilli V, Martinon F: The inflammasome, autoinflammatory diseases, and gout. Joint Bone Spine 2007, 74:571-576.

20. Kippen I, Klinenberg JR, Weinberger A, Wilcox WR: Factors affecting urate solubility in vitro. Ann Rheum Dis 1974, 33:313-317.

21. Loeb JN: The influence of temperature on the solubility of monosodium urate. Arthritis Rheum 1972, 15:189-192.
22. Wilcox WR, Khalaf AA: Nucleation of monosodium urate crystals. Ann Rheum Dis 1975, 34:332-339.

23. Muehleman C, Li J, Aigner T, Rappoport L, Mattson E, Hirschmugl C, Masuda K, Rosenthal AK: Association between crystals and cartilage degeneration in the ankle. J Rheumatol 2008, 35:1108-1117.

24. Pascual $E$, Ordonez S: Orderly arrayed deposit of urate crystals in gout suggest epitaxial formation. Ann Rheum Dis 1998, 57:255.

25. Simkin PA, Campbell PM, Larson EB: Gout in Heberden's nodes. Arthritis Rheum 1983, 26:94-97.

26. O'Dell JR: Gout in Heberden's nodes. Arthritis Rheum 1983, 26:1413-1414.

27. Parhami N, Greenstein N, Juozevicius JL: Erosive osteoarthritis and gout: gout in 36 joints. J Rheumatol 1986, 13:469-471.

28. Lally EV, Zimmermann B, Ho G Jr, Kaplan SR: Urate-mediated inflammation in nodal osteoarthritis: clinical and roentgenographic correlations. Arthritis Rheum 1989, 32:86-90.

29. Foldes K, Petersilge CA, Weisman MH, Resnick D: Nodal osteoarthritis and gout: a report of four new cases. Skeletal Radiol 1996, 25:421-424.

30. Fam AG, Stein J, Rubenstein J: Gouty arthritis in nodal osteoarthritis. Rheumatol 1996, 23:684-689.

31. Kawenoki-Minc E, Eyman E, Leo W, Werynska-Przybylska J: Osteoarthrosis and spondylosis in gouty patients. Analysis of 262 cases of gout. Reumatologia 1974, 12:267-267.

32. Choi HK, Atkinson K, Karlson EW, Curhan G: Obesity, weight change, hypertension, diuretic use, and risk of gout in men: the health professionals follow-up study. Arch Intern Med 2005, 165:742-748.

33. Oliveria SA, Felson DT, Cirillo PA, Reed JI, Walker AM: Body weight, body mass index, and incident symptomatic osteoarthritis of the hand, hip, and knee. Epidemiology 1999, 10:161-166.

34. Wright GD, Regan M, Deighton CM, Wallis G, Doherty M: Evidence for genetic anticipation in nodal osteoarthritis. Ann Rheum Dis 1998, 57:524-526.

35. Denoble AE, Huffman KM, Stabler TV, Kelly SJ, Hershfield MS, McDaniel GE, Coleman RE, Kraus VB: Uric acid is a danger signal of increasing risk for osteoarthritis through inflammasome activation. Proc Natl Acad Sci USA 2011, 108:2088-2093

36. Nowatzky J, Howard R, Pillinger MH, Krasnokutsky S: The role of uric acid and other crystals in osteoarthritis. Curr Rheumatol Rep 2010, 12:142-148.

37. Laurent TC: Solubility of Sodium Urate in the Presence of Chondroitin-4Sulphate. Nature 1964, 202:1334.

38. Katz WA, Schubert M: The interaction of monosodium urate with connective tissue components. J Clin Invest 1970, 49:1783-1789.

39. Burt HM, Dutt YC: Growth of monosodium urate monohydrate crystals: effect of cartilage and synovial fluid components on in vitro growth rates. Ann Rheum Dis 1986, 45:858-864.

40. Trivedi B, Marshall M, Belcher J, Roddy E: A systematic review of radiographic definitions of foot osteoarthritis in population-based studies. Osteoarthritis Cartilage 2010, 18:1027-1035.

41. Simkin PA: The pathogenesis of podagra. Ann Intern Med 1977, 86:230-233.

doi:10.1186/1757-1146-4-13

Cite this article as: Roddy: Revisiting the pathogenesis of podagra: why does gout target the foot? Journal of Foot and Ankle Research 2011 4:13.

\section{Submit your next manuscript to BioMed Central and take full advantage of:}

- Convenient online submission

- Thorough peer review

- No space constraints or color figure charges

- Immediate publication on acceptance

- Inclusion in PubMed, CAS, Scopus and Google Scholar

- Research which is freely available for redistribution 